\title{
Electronic Supplementary Information (ESI) Deep-Learning Architecture in QSPR Modeling for the Prediction of Energy Conversion Efficiency of Solar Cells
}

\author{
Jinkui Wu, ${ }^{a}$ Shibui Wang, ${ }^{a}$ Li Zhou, ${ }^{* a}$ Xu Ji, ${ }^{a}$ Yiyang Dai, ${ }^{a}$ Yagu Dang ${ }^{a}$ and Markus Kraft ${ }^{b c d}$ \\ ${ }^{a}$ School of Chemical Engineering, Sichuan University, Sichuan 610065, China. Email: chezli@scu.edu.cn \\ ${ }^{b}$ Cambridge Center for Advanced Research and Education in Singapore Ltd, 1 Create Way 138602, Singapore \\ ${ }^{c}$ Department of Chemical Engineering and Biotechnology, University of Cambridge, Philippa Farecett Drive, Cambridge CB3 \\ $O A S, U K$. \\ ${ }^{d}$ School of Chemical and Biomedical Engineering, Nanyang Technological University, 62 Nanyang Drive, Singapore 637459, \\ Singapore.
}

\section{DETAILED INFORMATION OF FRAGMENTS}
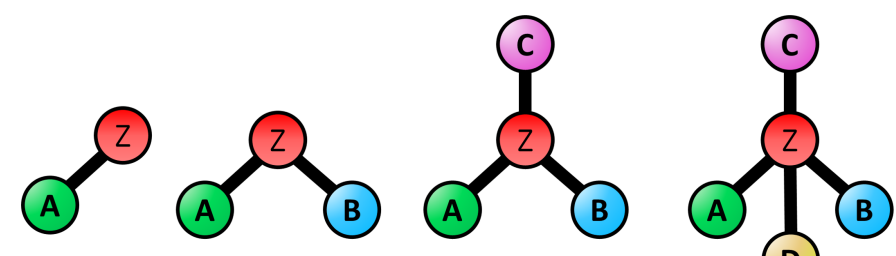

Figure S1. The probable sketch maps of atomic connection for a given fragments.

The molecular descriptor in this paper is based on molecular fragments. For a fragment, it contains a unique centric atom $\mathrm{Z}$ and at least one atom $(\mathrm{A}, \mathrm{B}, \mathrm{C}, \ldots)$ that connects with $\mathrm{Z}$. There are three types of chemical bonds between two connected atoms for molecules in CEPDB: single bond, aromatic bond, and double bond, and that for compounds in HOPV15 include another more: triple bond. The sketch map for a possible fragment is shown in Fig. S1. The detailed information for each fragment of organic compounds in CEPDB, NFADB and HOPV15 are shown in table S1, S2 and table $\mathrm{S} 3$ respectively. As is shown in the table $\mathrm{S} 1$, table $\mathrm{S} 2$ and table $\mathrm{S} 3$, the amount of the fragments in those three datasets are 56, 193, and 133 respectively.

Table S1. The detailed information of the fragments in CEPDB

\begin{tabular}{|c|c|c|c|c|c|c|c|c|c|}
\hline Index & Atom Z & Atom A & Bond & Atom B & Bond & Atom C & Bond & Atom D & Bond \\
\hline 1 & $\mathrm{C}$ (aromatic) & $\mathrm{C}$ (aromatic) & aromatic & $\mathrm{Se}$ (aromatic) & aromatic & $\mathrm{H}$ & single & - & - \\
\hline 2 & $\mathrm{C}$ (aromatic) & $\mathrm{C}$ (aromatic) & aromatic & $\mathrm{C}$ (aromatic) & aromatic & $\mathrm{H}$ & single & - & - \\
\hline 3 & $\mathrm{C}($ aromatic $)$ & $\mathrm{C}$ (aromatic) & aromatic & $\mathrm{C}$ (aromatic) & aromatic & $\mathrm{C}$ (aromatic) & aromatic & - & - \\
\hline 4 & $\mathrm{C}($ aromatic $)$ & $\mathrm{C}$ (aromatic) & aromatic & $\mathrm{O}$ (aromatic) & aromatic & $\mathrm{H}$ & single & - & - \\
\hline 5 & $\mathrm{O}$ (aromatic) & $\mathrm{C}$ (aromatic) & aromatic & $\mathrm{C}($ aromatic $)$ & aromatic & - & - & - & - \\
\hline 6 & $\mathrm{C}$ (aromatic) & $\mathrm{C}$ (aromatic) & aromatic & $\mathrm{C}($ aromatic $)$ & aromatic & $\mathrm{Si}$ & single & - & - \\
\hline 7 & $\mathrm{Si}$ & $\mathrm{C}$ (aromatic) & single & $\mathrm{C}$ & single & $\mathrm{H}$ & single & $\mathrm{H}$ & single \\
\hline 8 & $\mathrm{C}$ & $\mathrm{C}$ (aromatic) & single & Si & single & $\mathrm{C}$ & double & - & - \\
\hline 9 & $\mathrm{C}$ & $\mathrm{C}$ (aromatic) & single & $\mathrm{C}$ & double & $\mathrm{H}$ & single & - & - \\
\hline 10 & $\mathrm{C}($ aromatic $)$ & $\mathrm{C}$ (aromatic) & aromatic & $\mathrm{C}$ & single & $\mathrm{N}$ (aromatic) & aromatic & - & - \\
\hline 11 & $\mathrm{~N}$ (aromatic) & $\mathrm{C}$ (aromatic) & aromatic & $\mathrm{C}($ aromatic $)$ & aromatic & - & - & - & - \\
\hline 12 & $\mathrm{C}($ aromatic $)$ & $\mathrm{C}$ (aromatic) & aromatic & $\mathrm{N}$ (aromatic) & aromatic & $\mathrm{H}$ & single & - & - \\
\hline
\end{tabular}




\begin{tabular}{|c|c|c|c|c|c|c|c|c|c|}
\hline 13 & $\mathrm{C}$ (aromatic) & $\mathrm{C}$ (aromatic) & aromatic & $\mathrm{C}($ aromatic $)$ & aromatic & $\mathrm{Se}($ aromatic) & aromatic & - & - \\
\hline 14 & Se(aromatic) & $\mathrm{C}$ (aromatic) & aromatic & $\mathrm{C}$ (aromatic) & aromatic & - & - & - & - \\
\hline 15 & $\mathrm{C}($ aromatic $)$ & $\mathrm{C}$ (aromatic) & aromatic & $\mathrm{C}$ & single & $\mathrm{S}$ (aromatic) & aromatic & - & - \\
\hline 16 & $\mathrm{~S}($ aromatic $)$ & $\mathrm{C}$ (aromatic) & aromatic & $\mathrm{C}($ aromatic $)$ & aromatic & - & - & - & - \\
\hline 17 & $\mathrm{C}($ aromatic $)$ & $\mathrm{C}$ (aromatic) & aromatic & $\mathrm{S}$ (aromatic) & aromatic & $\mathrm{H}$ & single & - & - \\
\hline 18 & $\mathrm{C}$ (aromatic) & $\mathrm{C}$ (aromatic) & aromatic & $\mathrm{C}($ aromatic $)$ & aromatic & $\mathrm{C}$ & single & - & - \\
\hline 19 & $\mathrm{C}$ & $\mathrm{C}$ & single & $\mathrm{C}$ & double & $\mathrm{H}$ & single & - & - \\
\hline 20 & $\mathrm{C}$ & $\mathrm{C}$ (aromatic) & single & $\mathrm{C}$ & single & $\mathrm{H}$ & single & $\mathrm{H}$ & single \\
\hline 21 & $\mathrm{H}$ & $\mathrm{C}$ (aromatic) & single & - & - & - & - & - & - \\
\hline 22 & $\mathrm{H}$ & $\mathrm{Si}$ & single & - & - & - & - & - & - \\
\hline 23 & $\mathrm{H}$ & $\mathrm{C}$ & single & - & - & - & - & - & - \\
\hline 24 & $\mathrm{C}($ aromatic $)$ & $\mathrm{C}$ (aromatic) & aromatic & $\mathrm{C}($ aromatic $)$ & single & $\mathrm{Se}($ aromatic) & aromatic & - & - \\
\hline 25 & $\mathrm{C}$ (aromatic) & $\mathrm{C}$ (aromatic) & aromatic & $\mathrm{C}($ aromatic $)$ & aromatic & $\mathrm{C}$ & double & - & - \\
\hline 26 & $\mathrm{C}$ & $\mathrm{C}$ (aromatic) & double & $\mathrm{Si}$ & single & $\mathrm{H}$ & single & - & - \\
\hline 27 & $\mathrm{Si}$ & $\mathrm{C}$ & single & $\mathrm{C}$ & single & $\mathrm{H}$ & single & $\mathrm{H}$ & single \\
\hline 28 & $\mathrm{C}$ (aromatic) & $\mathrm{C}$ (aromatic) & aromatic & $\mathrm{C}$ (aromatic) & aromatic & $\mathrm{C}$ (aromatic) & single & - & - \\
\hline 29 & $\mathrm{~N}$ (aromatic) & $\mathrm{C}$ (aromatic) & aromatic & $\mathrm{C}($ aromatic $)$ & aromatic & $\mathrm{H}$ & single & - & - \\
\hline 30 & $\mathrm{C}$ (aromatic) & $\mathrm{C}$ (aromatic) & aromatic & $\mathrm{C}$ (aromatic) & single & $\mathrm{N}$ (aromatic) & aromatic & - & - \\
\hline 31 & $\mathrm{C}($ aromatic $)$ & $\mathrm{C}$ (aromatic) & aromatic & $\mathrm{C}$ (aromatic) & aromatic & $\mathrm{N}$ (aromatic) & aromatic & - & - \\
\hline 32 & $\mathrm{~N}$ (aromatic) & $\mathrm{C}$ (aromatic) & aromatic & $\mathrm{S}$ (aromatic) & aromatic & - & - & - & - \\
\hline 33 & $\mathrm{~S}($ aromatic) & $\mathrm{N}$ (aromatic) & aromatic & $\mathrm{N}$ (aromatic) & aromatic & - & - & - & - \\
\hline 34 & $\mathrm{C}$ (aromatic) & $\mathrm{C}$ (aromatic) & aromatic & $\mathrm{C}$ (aromatic) & aromatic & $\mathrm{O}$ (aromatic) & aromatic & - & - \\
\hline 35 & $\mathrm{C}$ (aromatic) & $\mathrm{C}$ (aromatic) & aromatic & $\mathrm{C}$ (aromatic) & single & $\mathrm{O}$ (aromatic) & aromatic & - & - \\
\hline 36 & $\mathrm{H}$ & $\mathrm{N}$ (aromatic) & single & - & - & - & - & - & - \\
\hline 37 & $\mathrm{C}$ (aromatic) & $\mathrm{C}$ (aromatic) & aromatic & $\mathrm{C}$ (aromatic) & single & $\mathrm{S}$ (aromatic) & aromatic & - & - \\
\hline 38 & $\mathrm{C}($ aromatic $)$ & $\mathrm{C}$ (aromatic) & aromatic & $\mathrm{C}$ (aromatic) & aromatic & $\mathrm{S}$ (aromatic) & aromatic & - & - \\
\hline 39 & $\mathrm{C}$ & $\mathrm{C}$ & single & $\mathrm{C}$ & single & $\mathrm{H}$ & single & $\mathrm{H}$ & single \\
\hline 40 & $\mathrm{C}$ & $\mathrm{C}$ (aromatic) & double & $\mathrm{C}$ & single & $\mathrm{H}$ & single & - & - \\
\hline 41 & $\mathrm{C}$ (aromatic) & $\mathrm{C}$ (aromatic) & aromatic & $\mathrm{C}$ & single & $\mathrm{Se}($ aromatic) & aromatic & - & - \\
\hline 42 & $\mathrm{C}$ & $\mathrm{Si}$ & single & $\mathrm{C}$ & double & $\mathrm{H}$ & single & - & - \\
\hline 43 & $\mathrm{C}$ & $\mathrm{C}$ (aromatic) & single & $\mathrm{C}$ & single & $\mathrm{C}$ & double & - & - \\
\hline 44 & $\mathrm{C}($ aromatic $)$ & $\mathrm{C}$ (aromatic) & single & $\mathrm{N}$ (aromatic) & aromatic & $\mathrm{N}$ (aromatic) & aromatic & - & - \\
\hline 45 & $\mathrm{C}$ (aromatic) & $\mathrm{N}$ (aromatic) & aromatic & $\mathrm{N}$ (aromatic) & aromatic & $\mathrm{H}$ & single & - & - \\
\hline 46 & $\mathrm{C}$ (aromatic) & $\mathrm{C}($ aromatic $)$ & single & $\mathrm{N}$ (aromatic) & aromatic & $\mathrm{S}$ (aromatic) & aromatic & - & - \\
\hline 47 & $\mathrm{C}$ & $\mathrm{C}($ aromatic $)$ & single & $\mathrm{C}$ (aromatic) & single & $\mathrm{H}$ & single & $\mathrm{H}$ & single \\
\hline 48 & $\mathrm{C}$ (aromatic) & $\mathrm{N}$ (aromatic) & aromatic & $\mathrm{S}$ (aromatic) & aromatic & $\mathrm{H}$ & single & - & - \\
\hline 49 & $\mathrm{C}$ & $\mathrm{Si}$ & single & $\mathrm{C}$ & single & $\mathrm{C}$ & double & - & - \\
\hline 50 & $\mathrm{C}$ & $\mathrm{C}$ & single & $\mathrm{C}$ & single & $\mathrm{C}$ & double & - & - \\
\hline 51 & $\mathrm{Si}$ & $\mathrm{C}$ (aromatic) & single & $\mathrm{C}$ (aromatic) & single & $\mathrm{H}$ & single & $\mathrm{H}$ & single \\
\hline 52 & $\mathrm{C}$ (aromatic) & $\mathrm{C}$ (aromatic) & aromatic & $\mathrm{O}$ (aromatic) & aromatic & $\mathrm{C}$ & single & - & - \\
\hline 53 & $\mathrm{C}$ (aromatic) & C & single & $\mathrm{N}$ (aromatic) & aromatic & $\mathrm{N}$ (aromatic) & aromatic & - & - \\
\hline 54 & $\mathrm{C}$ (aromatic) & $\mathrm{C}$ & single & $\mathrm{N}$ (aromatic) & aromatic & $\mathrm{S}$ (aromatic) & aromatic & - & - \\
\hline 55 & $\mathrm{C}$ & $\mathrm{N}$ (aromatic) & single & $\mathrm{H}$ & single & $\mathrm{H}$ & single & $\mathrm{H}$ & single \\
\hline 56 & $\mathrm{~N}$ (aromatic) & C(aromatic) & aromatic & $\mathrm{C}$ (aromatic) & aromatic & $\mathrm{C}$ & single & - & - \\
\hline
\end{tabular}


Table S2. The detailed information of the fragments in NFADB

\begin{tabular}{|c|c|c|c|c|c|c|c|c|c|}
\hline Index & Atom Z & Atom A & Bond & Atom B & Bond & Atom C & Bond & Atom D & Bond \\
\hline 1 & $\mathrm{C}$ & $\mathrm{N}$ & single & $\mathrm{H}$ & single & $\mathrm{H}$ & single & $\mathrm{H}$ & single \\
\hline 2 & $\mathrm{~N}$ & $\mathrm{C}$ & single & $\mathrm{C}$ & single & $\mathrm{C}$ & single & - & - \\
\hline 3 & $\mathrm{C}$ & $\mathrm{C}$ & single & $\mathrm{N}$ & single & $\mathrm{O}$ & double & - & - \\
\hline 4 & $\mathrm{O}$ & $\mathrm{C}$ & double & - & - & - & - & - & - \\
\hline 5 & $\mathrm{C}$ & $\mathrm{C}$ & single & $\mathrm{C}$ & single & $\mathrm{C}$ & double & - & - \\
\hline 6 & $\mathrm{C}$ & $\mathrm{C}$ & double & $\mathrm{C}($ aromatic $)$ & single & $\mathrm{H}$ & single & - & - \\
\hline 7 & $\mathrm{C}$ (aromatic) & $\mathrm{C}$ & single & $\mathrm{C}$ (aromatic) & aromatic & $\mathrm{O}$ (aromatic) & aromatic & - & - \\
\hline 8 & $\mathrm{O}$ (aromatic) & $\mathrm{C}$ (aromatic) & aromatic & $\mathrm{C}($ aromatic $)$ & aromatic & - & - & - & - \\
\hline 9 & $\mathrm{C}$ (aromatic) & $\mathrm{C}$ (aromatic) & aromatic & $\mathrm{O}$ (aromatic) & aromatic & $\mathrm{H}$ & single & - & - \\
\hline 10 & $\mathrm{C}$ (aromatic) & $\mathrm{C}$ (aromatic) & aromatic & $\mathrm{C}$ (aromatic) & aromatic & $\mathrm{H}$ & single & - & - \\
\hline 11 & $\mathrm{C}$ (aromatic) & $\mathrm{C}$ & single & $\mathrm{C}($ aromatic $)$ & aromatic & $\mathrm{C}$ (aromatic) & aromatic & - & - \\
\hline 12 & $\mathrm{C}$ & $\mathrm{O}$ & double & $\mathrm{C}($ aromatic $)$ & single & $\mathrm{C}$ (aromatic) & single & - & - \\
\hline 13 & $\mathrm{C}$ (aromatic) & $\mathrm{C}$ & single & $\mathrm{C}($ aromatic $)$ & aromatic & $\mathrm{S}$ (aromatic) & aromatic & - & - \\
\hline 14 & $\mathrm{C}$ (aromatic) & $\mathrm{C}$ (aromatic) & single & $\mathrm{C}$ (aromatic) & aromatic & $\mathrm{S}$ (aromatic) & aromatic & - & - \\
\hline 15 & $\mathrm{C}$ (aromatic) & $\mathrm{C}$ (aromatic) & single & $\mathrm{N}$ (aromatic) & aromatic & $\mathrm{N}$ (aromatic) & aromatic & - & - \\
\hline 16 & $\mathrm{~N}$ (aromatic) & $\mathrm{C}$ (aromatic) & aromatic & $\mathrm{C}$ (aromatic) & aromatic & - & - & - & - \\
\hline 17 & $\mathrm{C}$ (aromatic) & $\mathrm{C}$ (aromatic) & aromatic & $\mathrm{N}$ (aromatic) & aromatic & $\mathrm{H}$ & single & - & - \\
\hline 18 & $\mathrm{C}$ & $\mathrm{C}$ & single & $\mathrm{C}$ & double & $\mathrm{C}$ (aromatic) & single & - & - \\
\hline 19 & $\mathrm{C}$ & $\mathrm{C}$ & single & $\mathrm{C}$ & double & $\mathrm{H}$ & single & - & - \\
\hline 20 & $\mathrm{C}$ & $\mathrm{C}$ & single & $\mathrm{C}$ & single & $\mathrm{N}$ & single & $\mathrm{H}$ & single \\
\hline 21 & $\mathrm{~N}$ & $\mathrm{C}$ & single & $\mathrm{C}$ & double & - & - & - & - \\
\hline 22 & $\mathrm{C}$ & $\mathrm{C}$ & single & $\mathrm{N}$ & double & $\mathrm{F}$ & single & - & - \\
\hline 23 & $\mathrm{~F}$ & $\mathrm{C}$ & single & - & - & - & - & - & - \\
\hline 24 & $\mathrm{~S}$ (aromatic) & $\mathrm{C}$ (aromatic) & aromatic & $\mathrm{C}$ (aromatic) & aromatic & - & - & - & - \\
\hline 25 & $\mathrm{C}$ & $\mathrm{N}$ & single & $\mathrm{N}$ & single & $\mathrm{O}$ & double & - & - \\
\hline 26 & $\mathrm{H}$ & $\mathrm{C}$ & single & - & - & - & - & - & - \\
\hline 27 & $\mathrm{H}$ & $\mathrm{C}$ (aromatic) & single & - & - & - & - & - & - \\
\hline 28 & $\mathrm{C}$ (aromatic) & C & single & $\mathrm{N}$ (aromatic) & aromatic & $\mathrm{N}$ (aromatic) & aromatic & - & - \\
\hline 29 & $\mathrm{C}$ (aromatic) & $\mathrm{C}$ (aromatic) & single & $\mathrm{C}($ aromatic $)$ & aromatic & $\mathrm{N}$ (aromatic) & aromatic & - & - \\
\hline 30 & $\mathrm{C}$ (aromatic) & $\mathrm{C}$ & single & $\mathrm{C}$ (aromatic) & aromatic & $\mathrm{N}$ (aromatic) & aromatic & - & - \\
\hline 31 & $\mathrm{C}$ (aromatic) & $\mathrm{N}$ (aromatic) & aromatic & $\mathrm{N}$ (aromatic) & aromatic & $\mathrm{H}$ & single & - & - \\
\hline 32 & $\mathrm{~N}$ (aromatic) & $\mathrm{C}$ (aromatic) & aromatic & $\mathrm{N}$ (aromatic) & aromatic & - & - & - & - \\
\hline 33 & $\mathrm{C}$ (aromatic) & $\mathrm{C}$ (aromatic) & aromatic & $\mathrm{C}($ aromatic $)$ & aromatic & $\mathrm{F}$ & single & - & - \\
\hline 34 & $\mathrm{~F}$ & $\mathrm{C}$ (aromatic) & single & - & - & - & - & - & - \\
\hline 35 & $\mathrm{C}$ (aromatic) & $\mathrm{C}$ (aromatic) & single & $\mathrm{C}($ aromatic $)$ & aromatic & $\mathrm{C}$ (aromatic) & aromatic & - & - \\
\hline 36 & $\mathrm{C}$ (aromatic) & $\mathrm{C}$ (aromatic) & aromatic & $\mathrm{C}($ aromatic $)$ & aromatic & $\mathrm{S}$ (aromatic) & aromatic & - & - \\
\hline 37 & $\mathrm{C}$ & $\mathrm{N}$ & triple & $\mathrm{C}$ (aromatic) & single & - & - & - & - \\
\hline 38 & $\mathrm{~N}$ & $\mathrm{C}$ & triple & - & - & - & - & - & - \\
\hline 39 & $\mathrm{C}$ (aromatic) & $\mathrm{C}$ (aromatic) & aromatic & $\mathrm{C}($ aromatic $)$ & aromatic & $\mathrm{C}$ (aromatic) & aromatic & - & - \\
\hline 40 & $\mathrm{C}$ (aromatic) & $\mathrm{C}$ (aromatic) & aromatic & $\mathrm{C}($ aromatic $)$ & aromatic & $\mathrm{N}$ (aromatic) & aromatic & - & - \\
\hline 41 & $\mathrm{~N}$ (aromatic) & $\mathrm{C}$ (aromatic) & aromatic & $\mathrm{S}$ (aromatic) & aromatic & - & - & - & - \\
\hline 42 & $\mathrm{~S}$ (aromatic) & $\mathrm{N}$ (aromatic) & aromatic & $\mathrm{N}$ (aromatic) & aromatic & - & - & - & - \\
\hline 43 & $\mathrm{C}$ (aromatic) & $\mathrm{C}$ & single & $\mathrm{O}$ (aromatic) & aromatic & $\mathrm{N}$ (aromatic) & aromatic & - & - \\
\hline 44 & $\mathrm{C}$ (aromatic) & $\mathrm{C}$ (aromatic) & single & $\mathrm{C}$ (aromatic) & aromatic & $\mathrm{O}$ (aromatic) & aromatic & - & - \\
\hline 45 & $\mathrm{C}$ (aromatic) & $\mathrm{C}$ (aromatic) & aromatic & $\mathrm{S}$ (aromatic) & aromatic & $\mathrm{H}$ & single & - & - \\
\hline
\end{tabular}




\begin{tabular}{|c|c|c|c|c|c|c|c|c|c|}
\hline 46 & $\mathrm{C}($ aromatic) & $\mathrm{C}$ (aromatic) & aromatic & $\mathrm{C}$ (aromatic) & aromatic & $\mathrm{O}$ (aromatic) & aromatic & - & - \\
\hline 47 & $\mathrm{C}$ (aromatic) & $\mathrm{O}$ (aromatic) & aromatic & $\mathrm{N}$ (aromatic) & aromatic & $\mathrm{H}$ & single & - & - \\
\hline 48 & $\mathrm{C}$ & $\mathrm{O}$ & single & $\mathrm{O}$ & single & $\mathrm{C}$ (aromatic) & single & $\mathrm{C}$ (aromatic) & single \\
\hline 49 & $\mathrm{O}$ & $\mathrm{C}$ & single & $\mathrm{C}$ & single & - & - & - & - \\
\hline 50 & $\mathrm{C}$ & $\mathrm{C}$ & single & $\mathrm{O}$ & single & $\mathrm{H}$ & single & $\mathrm{H}$ & single \\
\hline 51 & $\mathrm{C}$ & $\mathrm{O}$ & single & $\mathrm{O}$ & double & $\mathrm{C}$ (aromatic) & single & - & - \\
\hline 52 & $\mathrm{O}$ & $\mathrm{C}$ & single & $\mathrm{H}$ & single & - & - & - & - \\
\hline 53 & $\mathrm{H}$ & $\mathrm{O}$ & single & - & - & - & - & - & - \\
\hline 54 & $\mathrm{C}$ (aromatic) & $\mathrm{C}$ (aromatic) & aromatic & $\mathrm{C}($ aromatic $)$ & aromatic & $\mathrm{Si}$ & single & - & - \\
\hline 55 & $\mathrm{Si}$ & $\mathrm{C}$ & single & $\mathrm{C}$ & single & $\mathrm{C}$ (aromatic) & single & $\mathrm{H}$ & single \\
\hline 56 & $\mathrm{C}$ & $\mathrm{C}$ & double & $\mathrm{H}$ & single & $\mathrm{Si}$ & single & - & - \\
\hline 57 & $\mathrm{C}$ & $\mathrm{C}$ & single & $\mathrm{C}$ & double & $\mathrm{O}$ & single & - & - \\
\hline 58 & $\mathrm{H}$ & $\mathrm{Si}$ & single & - & - & - & - & - & - \\
\hline 59 & $\mathrm{O}$ (aromatic) & $\mathrm{C}$ (aromatic) & aromatic & $\mathrm{N}$ (aromatic) & aromatic & - & - & - & - \\
\hline 60 & $\mathrm{~N}$ (aromatic) & $\mathrm{C}$ (aromatic) & aromatic & $\mathrm{O}$ (aromatic) & aromatic & - & - & - & - \\
\hline 61 & $\mathrm{C}($ aromatic $)$ & $\mathrm{C}$ (aromatic) & aromatic & $\mathrm{C}($ aromatic $)$ & aromatic & $S$ & single & - & - \\
\hline 62 & $S$ & $\mathrm{C}$ & single & $\mathrm{C}$ (aromatic) & single & - & - & - & - \\
\hline 63 & $\mathrm{C}$ & $\mathrm{C}$ & single & $\mathrm{H}$ & single & $\mathrm{H}$ & single & S & single \\
\hline 64 & $\mathrm{C}$ & $\mathrm{C}$ (aromatic) & single & $\mathrm{H}$ & single & $\mathrm{H}$ & single & $\mathrm{H}$ & single \\
\hline 65 & $\mathrm{C}$ & $\mathrm{C}$ & double & $\mathrm{C}$ (aromatic) & single & S & single & - & - \\
\hline 66 & $\mathrm{C}$ & $\mathrm{C}$ & single & $\mathrm{N}$ & triple & - & - & - & - \\
\hline 67 & $\mathrm{C}$ & $\mathrm{C}$ & double & $\mathrm{H}$ & single & $S$ & single & - & - \\
\hline 68 & S & $\mathrm{C}$ & single & $\mathrm{C}$ & single & - & - & - & - \\
\hline 69 & $\mathrm{C}$ & $\mathrm{C}$ & single & $\mathrm{C}$ & double & $S$ & single & - & - \\
\hline 70 & $\mathrm{C}$ (aromatic) & $\mathrm{C}$ (aromatic) & single & $\mathrm{O}$ (aromatic) & aromatic & $\mathrm{N}$ (aromatic) & aromatic & - & - \\
\hline 71 & $\mathrm{C}$ & $\mathrm{C}$ & double & $\mathrm{H}$ & single & $\mathrm{H}$ & single & - & - \\
\hline 72 & $\mathrm{C}$ (aromatic) & $\mathrm{O}$ & single & $\mathrm{C}$ (aromatic) & aromatic & $\mathrm{C}$ (aromatic) & aromatic & - & - \\
\hline 73 & $\mathrm{O}$ & $\mathrm{C}$ & single & $\mathrm{C}$ (aromatic) & single & - & - & - & - \\
\hline 74 & Si & $\mathrm{C}$ & single & $\mathrm{C}$ & single & $\mathrm{C}$ & single & $\mathrm{C}($ aromatic $)$ & single \\
\hline 75 & $\mathrm{C}$ (aromatic) & $\mathrm{N}$ (aromatic) & aromatic & $\mathrm{N}$ (aromatic) & aromatic & $\mathrm{Si}$ & single & - & - \\
\hline 76 & $\mathrm{C}$ (aromatic) & $\mathrm{C}$ (aromatic) & aromatic & $\mathrm{F}$ & single & $\mathrm{S}$ (aromatic) & aromatic & - & - \\
\hline 77 & $\mathrm{C}$ (aromatic) & $\mathrm{C}$ (aromatic) & aromatic & $\mathrm{N}$ (aromatic) & aromatic & $\mathrm{S}$ (aromatic) & aromatic & - & - \\
\hline 78 & $\mathrm{C}$ & $\mathrm{C}$ & single & $\mathrm{O}$ & single & $\mathrm{C}$ (aromatic) & single & $\mathrm{H}$ & single \\
\hline 79 & $\mathrm{C}$ (aromatic) & $\mathrm{O}$ & single & $\mathrm{C}$ (aromatic) & aromatic & $\mathrm{N}$ (aromatic) & aromatic & - & - \\
\hline 80 & $\mathrm{C}$ & $\mathrm{C}$ & double & $\mathrm{C}$ (aromatic) & single & $\mathrm{C}$ (aromatic) & single & - & - \\
\hline 81 & $\mathrm{C}($ aromatic $)$ & $\mathrm{C}($ aromatic $)$ & aromatic & $\mathrm{S}$ (aromatic) & aromatic & $\mathrm{Si}$ & single & - & - \\
\hline 82 & $\mathrm{C}$ & $\mathrm{C}$ & double & $\mathrm{N}$ & single & $S$ & single & - & - \\
\hline 83 & $\mathrm{~N}$ & $\mathrm{C}$ & single & $\mathrm{C}$ (aromatic) & single & $\mathrm{H}$ & single & - & - \\
\hline 84 & $\mathrm{C}($ aromatic $)$ & $\mathrm{N}$ & single & $\mathrm{C}($ aromatic $)$ & aromatic & $\mathrm{C}$ (aromatic) & aromatic & - & - \\
\hline 85 & $\mathrm{C}$ & $\mathrm{C}$ & single & $\mathrm{O}$ & double & $\mathrm{C}$ (aromatic) & single & - & - \\
\hline 86 & $\mathrm{H}$ & $\mathrm{N}$ & single & - & - & - & - & - & - \\
\hline 87 & $\mathrm{~N}$ & $\mathrm{C}$ & single & $\mathrm{C}$ & single & $\mathrm{C}$ (aromatic) & single & - & - \\
\hline 88 & $\mathrm{C}$ & $\mathrm{C}$ & single & $\mathrm{C}$ & double & $\mathrm{N}$ & single & - & - \\
\hline 89 & $\mathrm{C}$ & $\mathrm{C}$ & single & $\mathrm{C}$ & single & $\mathrm{C}$ & single & $\mathrm{C}$ (aromatic) & single \\
\hline 90 & $\mathrm{C}$ & $\mathrm{C}$ & single & $\mathrm{H}$ & single & $\mathrm{H}$ & single & $\mathrm{H}$ & single \\
\hline 91 & $\mathrm{C}$ & $\mathrm{C}$ (aromatic) & single & $\mathrm{C}($ aromatic $)$ & single & $S$ & single & S & single \\
\hline 92 & $\mathrm{C}$ & $\mathrm{C}$ & double & $\mathrm{N}$ & single & $\mathrm{C}$ (aromatic) & single & - & - \\
\hline
\end{tabular}




\begin{tabular}{|c|c|c|c|c|c|c|c|c|c|}
\hline 93 & $\mathrm{C}$ & $\mathrm{C}$ & double & $\mathrm{N}$ & single & $\mathrm{O}$ & single & & . \\
\hline 94 & $\mathrm{Si}$ & $\mathrm{C}$ & single & $\mathrm{C}$ & single & $\mathrm{C}$ (aromatic) & single & $\mathrm{C}($ aromatic $)$ & single \\
\hline 95 & $\mathrm{C}$ & $\mathrm{H}$ & single & $\mathrm{H}$ & single & $\mathrm{H}$ & single & $\mathrm{Si}$ & single \\
\hline 96 & $\mathrm{C}$ & $\mathrm{C}($ aromatic $)$ & single & F & single & $\mathrm{F}$ & single & $\mathrm{F}$ & single \\
\hline 97 & $\mathrm{C}$ & $\mathrm{C}$ & double & $\mathrm{O}$ & single & $\mathrm{C}$ (aromatic) & single & - & - \\
\hline 98 & $\mathrm{C}$ & $\mathrm{C}$ & double & $\mathrm{O}$ & single & $\mathrm{H}$ & single & - & - \\
\hline 99 & $\mathrm{C}$ & $\mathrm{N}$ (aromatic) & single & $\mathrm{H}$ & single & $\mathrm{H}$ & single & $\mathrm{H}$ & single \\
\hline 100 & $\mathrm{~N}$ (aromatic) & $\mathrm{C}$ & single & $\mathrm{C}($ aromatic $)$ & aromatic & $\mathrm{C}$ (aromatic) & aromatic & - & - \\
\hline 101 & $\mathrm{C}$ (aromatic) & $\mathrm{O}$ & double & $\mathrm{C}$ (aromatic) & aromatic & $\mathrm{N}$ (aromatic) & aromatic & - & - \\
\hline 102 & $\mathrm{O}$ & $\mathrm{C}$ (aromatic) & double & - & - & - & - & - & - \\
\hline 103 & $\mathrm{~N}$ (aromatic) & $\mathrm{C}$ (aromatic) & aromatic & $\mathrm{C}($ aromatic $)$ & aromatic & $\mathrm{C}$ (aromatic) & aromatic & - & - \\
\hline 104 & $\mathrm{C}$ & $\mathrm{C}$ & double & $\mathrm{C}$ (aromatic) & single & Si & single & - & - \\
\hline 105 & $\mathrm{Si}$ & $\mathrm{C}$ & single & $\mathrm{C}$ & single & $\mathrm{C}$ & single & $\mathrm{C}$ & single \\
\hline 106 & $\mathrm{C}$ & $\mathrm{C}$ & double & $\mathrm{N}$ & single & $\mathrm{H}$ & single & - & - \\
\hline 107 & $\mathrm{C}$ (aromatic) & $\mathrm{C}$ (aromatic) & single & $\mathrm{N}$ (aromatic) & aromatic & $\mathrm{S}$ (aromatic) & aromatic & - & - \\
\hline 108 & $\mathrm{C}$ (aromatic) & $\mathrm{C}$ (aromatic) & aromatic & $\mathrm{N}$ (aromatic) & aromatic & $\mathrm{F}$ & single & - & - \\
\hline 109 & $\mathrm{~N}$ & $\mathrm{C}$ (aromatic) & single & $S$ & double & - & - & - & - \\
\hline 110 & S & $\mathrm{N}$ & double & $\mathrm{N}$ & double & - & - & - & - \\
\hline 111 & $\mathrm{C}$ & $\mathrm{C}$ & single & $\mathrm{C}$ & single & $\mathrm{C}$ (aromatic) & single & $\mathrm{C}$ (aromatic) & single \\
\hline 112 & $\mathrm{C}$ (aromatic) & $\mathrm{N}$ (aromatic) & aromatic & $\mathrm{N}$ (aromatic) & aromatic & $\mathrm{N}$ (aromatic) & aromatic & - & - \\
\hline 113 & $\mathrm{C}$ & $\mathrm{C}$ & single & $\mathrm{O}$ & single & $\mathrm{O}$ & double & - & - \\
\hline 114 & $\mathrm{C}$ & $\mathrm{C}$ & single & $\mathrm{C}$ & double & $\mathrm{Si}$ & single & - & - \\
\hline 115 & $\mathrm{~N}$ (aromatic) & $\mathrm{C}($ aromatic $)$ & aromatic & $\mathrm{C}($ aromatic $)$ & aromatic & $\mathrm{H}$ & single & - & - \\
\hline 116 & $\mathrm{H}$ & $\mathrm{N}$ (aromatic) & single & - & - & - & - & - & - \\
\hline 117 & $\mathrm{C}$ (aromatic) & $\mathrm{C}$ (aromatic) & aromatic & $\mathrm{C}($ aromatic $)$ & aromatic & $\mathrm{N}$ (aromatic) & single & - & - \\
\hline 118 & $\mathrm{~N}$ (aromatic) & $\mathrm{C}$ (aromatic) & single & $\mathrm{C}($ aromatic $)$ & aromatic & $\mathrm{C}$ (aromatic) & aromatic & - & - \\
\hline 119 & $\mathrm{Si}$ & $\mathrm{C}$ (aromatic) & single & $\mathrm{C}($ aromatic $)$ & single & $\mathrm{C}$ (aromatic) & single & $\mathrm{H}$ & single \\
\hline 120 & $\mathrm{C}$ (aromatic) & $\mathrm{C}$ (aromatic) & aromatic & $\mathrm{O}$ (aromatic) & aromatic & $\mathrm{Si}$ & single & - & - \\
\hline 121 & $\mathrm{C}$ & $\mathrm{C}$ & double & $\mathrm{N}$ & single & $\mathrm{Si}$ & single & - & - \\
\hline 122 & $\mathrm{Si}$ & $\mathrm{C}$ & single & $\mathrm{C}($ aromatic $)$ & single & $\mathrm{C}$ (aromatic) & single & $\mathrm{H}$ & single \\
\hline 123 & $\mathrm{C}$ & $\mathrm{C}$ (aromatic) & single & $\mathrm{C}$ (aromatic) & double & $\mathrm{H}$ & single & - & - \\
\hline 124 & $\mathrm{C}$ (aromatic) & $\mathrm{C}$ & double & $\mathrm{C}($ aromatic $)$ & aromatic & $\mathrm{S}$ (aromatic) & aromatic & - & - \\
\hline 125 & $\mathrm{C}$ (aromatic) & $\mathrm{C}($ aromatic $)$ & double & $\mathrm{C}($ aromatic $)$ & aromatic & $\mathrm{S}$ (aromatic) & aromatic & - & - \\
\hline 126 & $\mathrm{C}$ & $\mathrm{C}$ & single & $\mathrm{C}($ aromatic $)$ & double & $\mathrm{H}$ & single & - & - \\
\hline 127 & $\mathrm{Si}$ & $\mathrm{C}$ (aromatic) & single & $\mathrm{C}($ aromatic $)$ & single & $\mathrm{C}$ (aromatic) & single & $\mathrm{C}$ (aromatic) & single \\
\hline 128 & $\mathrm{C}$ & $\mathrm{C}$ (aromatic) & single & $\mathrm{C}$ (aromatic) & single & $\mathrm{C}$ (aromatic) & single & $\mathrm{C}$ (aromatic) & single \\
\hline 129 & $\mathrm{C}$ (aromatic) & $\mathrm{C}$ (aromatic) & aromatic & $\mathrm{S}$ (aromatic) & aromatic & $\mathrm{S}$ (aromatic) & aromatic & - & - \\
\hline 130 & $\mathrm{C}$ (aromatic) & $\mathrm{C}$ (aromatic) & aromatic & $\mathrm{N}$ (aromatic) & aromatic & $\mathrm{Si}$ & single & - & - \\
\hline 131 & $\mathrm{C}$ & $\mathrm{C}$ & double & $\mathrm{Si}$ & single & $S$ & single & - & - \\
\hline 132 & $\mathrm{C}$ & $\mathrm{C}$ & double & $\mathrm{O}$ & single & $\mathrm{Si}$ & single & - & - \\
\hline 133 & $\mathrm{C}$ & $\mathrm{C}$ & single & F & single & F & single & $\mathrm{F}$ & single \\
\hline 134 & $\mathrm{C}$ (aromatic) & $\mathrm{N}$ (aromatic) & single & $\mathrm{N}$ (aromatic) & aromatic & $\mathrm{N}$ (aromatic) & aromatic & - & - \\
\hline 135 & $\mathrm{C}$ (aromatic) & $\mathrm{C}$ (aromatic) & aromatic & $\mathrm{N}$ (aromatic) & single & $\mathrm{N}$ (aromatic) & aromatic & - & - \\
\hline 136 & $\mathrm{~N}$ & $\mathrm{C}$ & single & $\mathrm{C}($ aromatic) & single & $\mathrm{C}$ (aromatic) & single & - & - \\
\hline 137 & $\mathrm{C}$ (aromatic) & $\mathrm{N}$ & single & $\mathrm{N}$ (aromatic) & aromatic & $\mathrm{N}$ (aromatic) & aromatic & - & - \\
\hline 138 & Si & $\mathrm{C}$ & single & $\mathrm{C}$ & single & $\mathrm{C}$ & single & $\mathrm{H}$ & single \\
\hline 139 & $\mathrm{C}$ & $\mathrm{C}$ & single & $\mathrm{C}($ aromatic $)$ & single & $\mathrm{C}$ (aromatic) & double & - & - \\
\hline
\end{tabular}




\begin{tabular}{|c|c|c|c|c|c|c|c|c|c|}
\hline 140 & $\mathrm{C}$ & $\mathrm{C}$ & single & $\mathrm{C}$ & single & $\mathrm{O}$ & double & . & - \\
\hline 141 & $\mathrm{C}$ & $\mathrm{C}$ & single & $\mathrm{C}$ & single & $\mathrm{O}$ & single & $\mathrm{H}$ & single \\
\hline 142 & $\mathrm{C}$ (aromatic) & $\mathrm{C}$ & single & $\mathrm{N}$ (aromatic) & aromatic & $\mathrm{S}$ (aromatic) & aromatic & - & - \\
\hline 143 & $\mathrm{C}$ & $\mathrm{C}($ aromatic $)$ & double & $\mathrm{H}$ & single & $\mathrm{H}$ & single & - & - \\
\hline 144 & $\mathrm{C}$ (aromatic) & $\mathrm{O}$ & double & $\mathrm{C}$ (aromatic) & aromatic & $\mathrm{C}$ (aromatic) & aromatic & - & - \\
\hline 145 & $\mathrm{C}$ (aromatic) & $\mathrm{C}$ & double & $\mathrm{C}($ aromatic $)$ & aromatic & $\mathrm{N}$ (aromatic) & aromatic & - & - \\
\hline 146 & $\mathrm{C}$ & $\mathrm{N}$ & single & S & single & S & double & - & - \\
\hline 147 & $S$ & $\mathrm{C}$ & double & - & - & - & - & - & - \\
\hline 148 & $\mathrm{C}$ & $\mathrm{N}$ & single & $\mathrm{O}$ & double & $\mathrm{C}$ (aromatic) & single & - & - \\
\hline 149 & $\mathrm{C}($ aromatic $)$ & $\mathrm{N}$ & single & $\mathrm{C}($ aromatic $)$ & aromatic & $\mathrm{N}$ (aromatic) & aromatic & - & - \\
\hline 150 & $\mathrm{C}$ & $\mathrm{C}$ & single & $\mathrm{C}$ (aromatic) & single & $\mathrm{H}$ & single & S & single \\
\hline 151 & $\mathrm{~N}$ & $\mathrm{C}$ (aromatic) & single & $\mathrm{H}$ & single & $\mathrm{H}$ & single & - & - \\
\hline 152 & $\mathrm{C}$ & $\mathrm{C}$ & single & $\mathrm{O}$ & single & $\mathrm{H}$ & single & $\mathrm{Si}$ & single \\
\hline 153 & $\mathrm{C}$ & $\mathrm{C}$ & double & $\mathrm{N}$ & single & $\mathrm{N}$ (aromatic) & single & - & - \\
\hline 154 & $\mathrm{C}$ & $\mathrm{C}$ & double & $\mathrm{N}$ (aromatic) & single & $\mathrm{H}$ & single & - & - \\
\hline 155 & $\mathrm{C}$ & $\mathrm{O}$ & double & $\mathrm{Si}$ & single & $\mathrm{Si}$ & single & - & - \\
\hline 156 & $\mathrm{C}$ & $\mathrm{F}$ & single & $\mathrm{F}$ & single & $\mathrm{F}$ & single & $\mathrm{Si}$ & single \\
\hline 157 & $\mathrm{C}$ (aromatic) & $\mathrm{C}($ aromatic $)$ & aromatic & $\mathrm{N}$ (aromatic) & single & $\mathrm{S}$ (aromatic) & aromatic & - & - \\
\hline 158 & $\mathrm{C}$ (aromatic) & $\mathrm{O}$ (aromatic) & aromatic & $\mathrm{N}$ (aromatic) & single & $\mathrm{N}$ (aromatic) & aromatic & - & - \\
\hline 159 & $\mathrm{C}$ (aromatic) & $\mathrm{O}$ & single & $\mathrm{C}($ aromatic $)$ & aromatic & $\mathrm{S}$ (aromatic) & aromatic & - & - \\
\hline 160 & $\mathrm{C}$ & $\mathrm{C}$ & single & $\mathrm{C}$ & single & $\mathrm{H}$ & single & $S$ & single \\
\hline 161 & $\mathrm{C}$ (aromatic) & $\mathrm{N}$ & single & $\mathrm{C}$ (aromatic) & aromatic & $\mathrm{O}$ (aromatic) & aromatic & - & - \\
\hline 162 & $\mathrm{C}$ & $\mathrm{N}$ & triple & $\mathrm{Si}$ & single & - & - & - & - \\
\hline 163 & Si & $\mathrm{C}$ & single & $\mathrm{C}($ aromatic $)$ & single & $\mathrm{C}$ (aromatic) & single & $\mathrm{C}$ (aromatic) & single \\
\hline 164 & $\mathrm{C}$ & $\mathrm{C}$ & triple & $\mathrm{C}$ (aromatic) & single & - & - & - & - \\
\hline 165 & $\mathrm{C}$ & $\mathrm{C}$ & single & $\mathrm{C}($ aromatic $)$ & single & $\mathrm{C}$ (aromatic) & single & $\mathrm{C}$ (aromatic) & single \\
\hline 166 & $\mathrm{C}$ & $\mathrm{C}$ & double & S & single & S & single & - & - \\
\hline 167 & $\mathrm{C}$ (aromatic) & $\mathrm{N}$ (aromatic) & aromatic & $\mathrm{S}$ (aromatic) & aromatic & $\mathrm{Si}$ & single & - & - \\
\hline 168 & $\mathrm{C}$ (aromatic) & $\mathrm{O}$ & double & $\mathrm{C}$ (aromatic) & aromatic & $\mathrm{O}$ (aromatic) & aromatic & - & - \\
\hline 169 & $\mathrm{C}$ (aromatic) & $\mathrm{C}($ aromatic $)$ & aromatic & $\mathrm{O}$ (aromatic) & aromatic & $\mathrm{N}$ (aromatic) & single & - & - \\
\hline 170 & $\mathrm{C}$ & $\mathrm{C}$ & double & $\mathrm{N}$ (aromatic) & single & S & single & - & - \\
\hline 171 & $\mathrm{C}$ & $\mathrm{C}$ & single & $\mathrm{C}$ & single & $\mathrm{C}$ & single & $\mathrm{O}$ & single \\
\hline 172 & $\mathrm{C}$ & $\mathrm{O}$ & double & $\mathrm{N}$ (aromatic) & single & $\mathrm{N}$ (aromatic) & single & - & - \\
\hline 173 & $\mathrm{C}$ (aromatic) & $\mathrm{N}$ & single & $\mathrm{O}$ (aromatic) & aromatic & $\mathrm{N}$ (aromatic) & aromatic & - & - \\
\hline 174 & $\mathrm{C}$ & $\mathrm{C}$ & double & $\mathrm{N}$ & single & $\mathrm{N}$ & single & - & - \\
\hline 175 & $\mathrm{C}($ aromatic $)$ & $\mathrm{N}$ & single & $\mathrm{C}$ (aromatic) & aromatic & $\mathrm{S}$ (aromatic) & aromatic & - & - \\
\hline 176 & $\mathrm{~N}$ & $\mathrm{C}$ & single & $\mathrm{C}$ (aromatic) & single & $\mathrm{Si}$ & single & - & - \\
\hline 177 & Si & $\mathrm{C}$ & single & $\mathrm{C}$ & single & $\mathrm{N}$ & single & $\mathrm{C}($ aromatic $)$ & single \\
\hline 178 & $\mathrm{C}$ (aromatic) & $\mathrm{O}$ (aromatic) & aromatic & $\mathrm{N}$ (aromatic) & aromatic & $\mathrm{Si}$ & single & - & - \\
\hline 179 & $\mathrm{C}$ & $\mathrm{C}$ & double & $\mathrm{O}$ & single & $\mathrm{N}$ (aromatic) & single & - & - \\
\hline 180 & $\mathrm{C}$ & $\mathrm{C}$ & single & $\mathrm{C}$ & double & $\mathrm{N}$ (aromatic) & single & - & - \\
\hline 181 & $\mathrm{C}$ & $\mathrm{C}$ & single & $\mathrm{H}$ & single & $\mathrm{Si}$ & single & $S$ & single \\
\hline 182 & $\mathrm{C}$ (aromatic) & $\mathrm{C}$ & double & $\mathrm{N}$ (aromatic) & aromatic & $\mathrm{S}$ (aromatic) & aromatic & - & - \\
\hline 183 & $\mathrm{C}$ & $\mathrm{C}$ & single & $\mathrm{C}$ & single & $\mathrm{C}$ (aromatic) & double & - & - \\
\hline 184 & $\mathrm{C}$ & $\mathrm{C}$ & single & $\mathrm{C}$ & single & $\mathrm{C}$ & single & S & single \\
\hline 185 & $\mathrm{C}$ & $\mathrm{O}$ & single & $\mathrm{O}$ & double & Si & single & - & - \\
\hline 186 & $\mathrm{C}$ & $\mathrm{C}$ (aromatic) & double & $\mathrm{H}$ & single & $\mathrm{Si}$ & single & - & - \\
\hline
\end{tabular}




\begin{tabular}{|c|c|c|c|c|c|c|c|c|c|}
\hline 187 & $\mathrm{C}$ & $\mathrm{C}$ & single & $\mathrm{C}$ & triple & - & - & - & - \\
\hline 188 & $\mathrm{C}$ & $\mathrm{C}$ & triple & $\mathrm{Si}$ & single & - & - & - & - \\
\hline 189 & $\mathrm{C}$ & $\mathrm{O}$ & double & $\mathrm{C}($ aromatic $)$ & single & $\mathrm{H}$ & single & - & - \\
\hline 190 & $\mathrm{Si}$ & $\mathrm{C}$ & single & $\mathrm{C}$ & single & $\mathrm{C}$ & single & $\mathrm{N}$ & single \\
\hline 191 & $\mathrm{~N}$ & $\mathrm{C}$ & single & $\mathrm{C}$ & single & $\mathrm{Si}$ & single & - & - \\
\hline 192 & $\mathrm{C}$ & $\mathrm{C}$ (aromatic) & single & $\mathrm{C}$ (aromatic) & single & $\mathrm{C}$ (aromatic) & single & $\mathrm{H}$ & single \\
\hline 193 & $\mathrm{C}$ & $\mathrm{C}$ & single & $\mathrm{C}($ aromatic $)$ & single & $\mathrm{C}$ (aromatic) & single & $\mathrm{H}$ & single \\
\hline
\end{tabular}

Table S3. The detailed information of the fragments in HOPV15

\begin{tabular}{|c|c|c|c|c|c|c|c|c|c|}
\hline Index & Atom Z & Atom A & Bond & Atom B & Bond & Atom C & Bond & Atom D & Bond \\
\hline 1 & $\mathrm{C}$ & $\mathrm{C}$ (aromatic) & single & $\mathrm{H}$ & single & $\mathrm{H}$ & single & $\mathrm{H}$ & single \\
\hline 2 & $\mathrm{C}($ aromatic $)$ & $\mathrm{C}$ & single & $\mathrm{C}$ (aromatic) & aromatic & $\mathrm{S}$ (aromatic) & aromatic & - & - \\
\hline 3 & $\mathrm{C}($ aromatic $)$ & $\mathrm{C}($ aromatic $)$ & aromatic & $\mathrm{C}$ (aromatic) & aromatic & $\mathrm{H}$ & single & - & - \\
\hline 4 & $\mathrm{C}$ (aromatic) & $\mathrm{C}$ (aromatic) & single & $\mathrm{C}$ (aromatic) & aromatic & $\mathrm{S}$ (aromatic) & aromatic & - & - \\
\hline 5 & $\mathrm{C}$ (aromatic) & $\mathrm{C}$ (aromatic) & single & $\mathrm{C}$ (aromatic) & aromatic & $\mathrm{C}$ (aromatic) & aromatic & - & - \\
\hline 6 & $\mathrm{C}$ (aromatic) & $\mathrm{C}$ (aromatic) & aromatic & $\mathrm{C}$ (aromatic) & aromatic & $\mathrm{C}$ (aromatic) & aromatic & - & - \\
\hline 7 & $\mathrm{~S}$ (aromatic) & $\mathrm{C}$ (aromatic) & aromatic & $\mathrm{C}$ (aromatic) & aromatic & No & - & - & - \\
\hline 8 & $\mathrm{C}($ aromatic $)$ & $\mathrm{C}($ aromatic $)$ & aromatic & $\mathrm{S}$ (aromatic) & aromatic & $\mathrm{H}$ & single & - & - \\
\hline 9 & $\mathrm{C}($ aromatic $)$ & $\mathrm{C}($ aromatic $)$ & aromatic & $\mathrm{S}$ (aromatic) & aromatic & S & single & - & - \\
\hline 10 & S & $\mathrm{C}$ & single & $\mathrm{C}$ (aromatic) & single & $\mathrm{O}$ & double & $\mathrm{O}$ & double \\
\hline 11 & $\mathrm{C}$ & $S$ & single & $\mathrm{H}$ & single & $\mathrm{H}$ & single & $\mathrm{H}$ & single \\
\hline 12 & $\mathrm{O}$ & $S$ & double & - & - & $\mathrm{No}$ & - & - & - \\
\hline 13 & $\mathrm{C}$ (aromatic) & $\mathrm{C}$ (aromatic) & aromatic & $\mathrm{C}$ (aromatic) & aromatic & $\mathrm{S}$ (aromatic) & aromatic & - & - \\
\hline 14 & $\mathrm{H}$ & $\mathrm{C}$ & single & - & - & No & - & - & - \\
\hline 15 & $\mathrm{H}$ & $\mathrm{C}($ aromatic $)$ & single & - & - & No & - & - & - \\
\hline 16 & $\mathrm{C}$ & $\mathrm{H}$ & single & $\mathrm{H}$ & single & $\mathrm{H}$ & single & $\mathrm{N}$ & single \\
\hline 17 & $\mathrm{~N}$ & $\mathrm{C}$ & single & $\mathrm{C}$ & single & $\mathrm{C}$ (aromatic) & single & - & - \\
\hline 18 & $\mathrm{C}$ (aromatic) & $\mathrm{C}$ (aromatic) & aromatic & $\mathrm{C}$ (aromatic) & aromatic & $\mathrm{N}$ & single & - & - \\
\hline 19 & $\mathrm{C}$ (aromatic) & $\mathrm{C}$ & single & $\mathrm{C}$ (aromatic) & aromatic & $\mathrm{C}$ (aromatic) & aromatic & - & - \\
\hline 20 & $\mathrm{C}$ & $\mathrm{C}$ & single & $\mathrm{C}$ & double & $\mathrm{C}$ (aromatic) & single & - & - \\
\hline 21 & $\mathrm{C}$ & $\mathrm{C}$ & single & $\mathrm{O}$ & double & $\mathrm{N}$ & single & - & - \\
\hline 22 & $\mathrm{O}$ & $\mathrm{C}$ & double & - & - & $\mathrm{No}$ & - & - & - \\
\hline 23 & $\mathrm{C}$ (aromatic) & $\mathrm{C}$ (aromatic) & aromatic & $\mathrm{C}$ (aromatic) & aromatic & $\mathrm{N}$ (aromatic) & aromatic & - & - \\
\hline 24 & $\mathrm{~N}$ (aromatic) & $\mathrm{C}($ aromatic $)$ & aromatic & $\mathrm{S}$ (aromatic) & aromatic & No & - & - & - \\
\hline 25 & $\mathrm{~S}$ (aromatic) & $\mathrm{N}$ (aromatic) & aromatic & $\mathrm{N}$ (aromatic) & aromatic & No & - & - & - \\
\hline 26 & $\mathrm{C}$ & $\mathrm{H}$ & single & $\mathrm{H}$ & single & $\mathrm{H}$ & single & $\mathrm{N}$ (aromatic) & single \\
\hline 27 & $\mathrm{~N}$ (aromatic) & $\mathrm{C}$ & single & $\mathrm{C}$ (aromatic) & aromatic & $\mathrm{C}$ (aromatic) & aromatic & - & - \\
\hline 28 & $\mathrm{C}$ & $\mathrm{C}$ & double & $\mathrm{C}$ (aromatic) & single & $\mathrm{Si}$ & single & - & - \\
\hline 29 & $\mathrm{Si}$ & $\mathrm{C}$ & single & $\mathrm{C}$ & single & $\mathrm{C}$ & single & $\mathrm{C}$ & single \\
\hline 30 & $\mathrm{C}$ & $\mathrm{H}$ & single & $\mathrm{H}$ & single & $\mathrm{H}$ & single & $\mathrm{Si}$ & single \\
\hline 31 & $\mathrm{C}$ (aromatic) & $\mathrm{C}$ (aromatic) & aromatic & $\mathrm{C}$ (aromatic) & aromatic & $\mathrm{F}$ & single & - & - \\
\hline 32 & $\mathrm{~F}$ & $\mathrm{C}$ (aromatic) & single & - & - & No & - & - & - \\
\hline 33 & $\mathrm{C}$ & $\mathrm{C}$ & triple & $\mathrm{H}$ & single & No & - & - & - \\
\hline 34 & $\mathrm{C}$ & $\mathrm{C}$ & triple & $\mathrm{C}$ (aromatic) & single & No & - & - & - \\
\hline 35 & $\mathrm{C}$ (aromatic) & $\mathrm{C}$ (aromatic) & aromatic & $\mathrm{C}$ (aromatic) & aromatic & $\mathrm{O}$ & single & - & - \\
\hline 36 & $\mathrm{O}$ & $\mathrm{C}$ & single & $\mathrm{C}$ (aromatic) & single & No & - & - & - \\
\hline
\end{tabular}




\begin{tabular}{|c|c|c|c|c|c|c|c|c|c|}
\hline 37 & $\mathrm{C}$ & $\mathrm{O}$ & single & $\mathrm{H}$ & single & $\mathrm{H}$ & single & $\mathrm{H}$ & single \\
\hline 38 & $\mathrm{~N}$ (aromatic) & $\mathrm{C}$ (aromatic) & aromatic & $\mathrm{C}$ (aromatic) & aromatic & No & - & - & - \\
\hline 39 & $\mathrm{C}$ (aromatic) & $\mathrm{C}($ aromatic $)$ & single & $\mathrm{C}$ (aromatic) & aromatic & $\mathrm{N}$ (aromatic) & aromatic & - & - \\
\hline 40 & $\mathrm{C}$ & $\mathrm{C}$ & single & $\mathrm{H}$ & single & $\mathrm{H}$ & single & $\mathrm{H}$ & single \\
\hline 41 & $\mathrm{C}$ & $\mathrm{C}$ & single & $\mathrm{C}$ & single & $\mathrm{C}$ & double & - & - \\
\hline 42 & $\mathrm{C}$ & $\mathrm{C}$ & single & $\mathrm{C}$ & double & $\mathrm{H}$ & single & - & - \\
\hline 43 & $\mathrm{C}$ & $\mathrm{C}$ & single & $\mathrm{C}$ (aromatic) & single & S & double & - & - \\
\hline 44 & $\mathrm{C}$ (aromatic) & $\mathrm{C}$ (aromatic) & single & $\mathrm{S}$ (aromatic) & aromatic & $\mathrm{N}$ (aromatic) & aromatic & - & - \\
\hline 45 & $\mathrm{C}$ (aromatic) & $\mathrm{C}$ & single & $\mathrm{C}$ (aromatic) & aromatic & $\mathrm{N}$ (aromatic) & aromatic & - & - \\
\hline 46 & S & $\mathrm{C}$ & double & $\mathrm{C}$ & double & No & - & - & - \\
\hline 47 & $\mathrm{C}$ & $\mathrm{C}$ & single & $S$ & double & $\mathrm{H}$ & single & - & - \\
\hline 48 & $\mathrm{~N}$ & $\mathrm{C}$ & single & $\mathrm{C}$ & single & $\mathrm{C}$ & single & - & - \\
\hline 49 & $\mathrm{C}$ & $\mathrm{C}$ & double & $\mathrm{C}$ (aromatic) & single & $\mathrm{N}$ & single & - & - \\
\hline 50 & $\mathrm{C}$ & $\mathrm{C}$ (aromatic) & single & $\mathrm{F}$ & single & $\mathrm{F}$ & single & $\mathrm{F}$ & single \\
\hline 51 & $\mathrm{~F}$ & C & single & - & - & No & - & - & - \\
\hline 52 & $\mathrm{C}$ & $\mathrm{C}$ & single & $\mathrm{C}$ & single & $\mathrm{C}($ aromatic $)$ & single & $\mathrm{C}$ (aromatic) & single \\
\hline 53 & $\mathrm{~N}$ & $\mathrm{C}($ aromatic $)$ & single & $\mathrm{C}$ (aromatic) & single & $\mathrm{C}($ aromatic $)$ & single & - & - \\
\hline 54 & $\mathrm{C}$ & $\mathrm{C}$ & double & $\mathrm{C}$ (aromatic) & single & $\mathrm{H}$ & single & - & - \\
\hline 55 & $\mathrm{C}$ & $\mathrm{C}$ & single & $\mathrm{N}$ & triple & No & - & - & - \\
\hline 56 & $\mathrm{~N}$ & $\mathrm{C}$ & triple & - & - & No & - & - & - \\
\hline 57 & $\mathrm{C}$ & $\mathrm{C}$ & single & $\mathrm{O}$ & single & $\mathrm{O}$ & double & - & - \\
\hline 58 & $\mathrm{O}$ & $\mathrm{C}$ & single & $\mathrm{H}$ & single & No & - & - & - \\
\hline 59 & $\mathrm{H}$ & $\mathrm{O}$ & single & - & - & No & - & - & - \\
\hline 60 & $\mathrm{C}$ & $\mathrm{C}$ & double & $\mathrm{H}$ & single & $\mathrm{H}$ & single & - & - \\
\hline 61 & $\mathrm{C}$ & $\mathrm{C}$ & single & $\mathrm{C}$ & double & S & single & - & - \\
\hline 62 & S & $\mathrm{C}$ & single & $\mathrm{C}$ & single & No & - & - & - \\
\hline 63 & $\mathrm{C}$ & S & single & S & double & $\mathrm{N}$ & single & - & - \\
\hline 64 & $S$ & $\mathrm{C}$ & double & - & - & No & - & - & - \\
\hline 65 & $\mathrm{C}$ & $\mathrm{C}$ & single & $\mathrm{H}$ & single & $\mathrm{H}$ & single & $\mathrm{N}$ & single \\
\hline 66 & $\mathrm{C}$ (aromatic) & $\mathrm{C}$ (aromatic) & aromatic & $\mathrm{H}$ & single & $\mathrm{Se}$ (aromatic) & aromatic & - & - \\
\hline 67 & $\mathrm{Se}($ aromatic) & $\mathrm{C}$ (aromatic) & aromatic & $\mathrm{C}$ (aromatic) & aromatic & No & - & - & - \\
\hline 68 & $\mathrm{C}$ & $\mathrm{C}$ & single & $\mathrm{C}$ & single & $\mathrm{C}$ & single & $\mathrm{C}($ aromatic $)$ & single \\
\hline 69 & $\mathrm{C}$ & $\mathrm{C}$ (aromatic) & single & $\mathrm{C}$ (aromatic) & single & $\mathrm{C}$ (aromatic) & single & $\mathrm{C}$ (aromatic) & single \\
\hline 70 & $\mathrm{C}$ & $\mathrm{C}$ & single & $\mathrm{C}$ (aromatic) & single & $\mathrm{O}$ & double & - & - \\
\hline 71 & $\mathrm{~N}$ (aromatic) & $\mathrm{C}$ (aromatic) & single & $\mathrm{C}$ (aromatic) & aromatic & $\mathrm{C}$ (aromatic) & aromatic & - & - \\
\hline 72 & $\mathrm{C}$ (aromatic) & $\mathrm{C}$ (aromatic) & aromatic & $\mathrm{C}$ (aromatic) & aromatic & $\mathrm{N}$ (aromatic) & single & - & - \\
\hline 73 & $\mathrm{C}$ & $\mathrm{C}$ & double & $\mathrm{C}$ (aromatic) & single & S & single & - & - \\
\hline 74 & S & $\mathrm{C}$ & single & $\mathrm{C}$ & single & $\mathrm{O}$ & double & $\mathrm{O}$ & double \\
\hline 75 & $\mathrm{C}$ & $\mathrm{C}$ & triple & $\mathrm{Si}$ & single & No & - & - & - \\
\hline 76 & $\mathrm{C}$ (aromatic) & $\mathrm{C}$ (aromatic) & aromatic & $\mathrm{S}$ (aromatic) & aromatic & $\mathrm{N}$ (aromatic) & aromatic & - & - \\
\hline 77 & $\mathrm{C}$ & $\mathrm{C}$ & single & $\mathrm{C}$ & single & S & double & - & - \\
\hline 78 & $\mathrm{~N}$ & $\mathrm{C}$ (aromatic) & single & $\mathrm{O}$ & single & $\mathrm{O}$ & double & - & - \\
\hline 79 & $\mathrm{O}$ & $\mathrm{N}$ & double & - & - & No & - & - & - \\
\hline 80 & $\mathrm{O}$ & $\mathrm{N}$ & single & - & - & No & - & - & - \\
\hline 81 & $\mathrm{C}$ & $\mathrm{C}$ (aromatic) & single & $\mathrm{O}$ & single & $\mathrm{O}$ & double & - & - \\
\hline 82 & $\mathrm{O}$ & $\mathrm{C}$ & single & $\mathrm{C}$ & single & No & - & - & - \\
\hline 83 & $\mathrm{C}$ (aromatic) & $\mathrm{C}$ (aromatic) & aromatic & $\mathrm{C}$ (aromatic) & aromatic & $\mathrm{Si}$ & single & - & - \\
\hline
\end{tabular}




\begin{tabular}{|c|c|c|c|c|c|c|c|c|c|}
\hline 84 & $\mathrm{Si}$ & $\mathrm{C}$ & single & $\mathrm{C}$ & single & $\mathrm{C}($ aromatic $)$ & single & $\mathrm{C}($ aromatic $)$ & single \\
\hline 85 & $\mathrm{C}($ aromatic $)$ & $\mathrm{C}$ (aromatic) & aromatic & $\mathrm{H}$ & single & $\mathrm{N}$ (aromatic) & aromatic & - & - \\
\hline 86 & $\mathrm{C}$ & $\mathrm{C}$ (aromatic) & single & $\mathrm{O}$ & single & $\mathrm{H}$ & single & $\mathrm{H}$ & single \\
\hline 87 & $\mathrm{C}$ & $\mathrm{C}$ (aromatic) & single & $\mathrm{O}$ & double & $\mathrm{N}$ & single & - & - \\
\hline 88 & $\mathrm{C}$ & $\mathrm{C}$ & single & $\mathrm{C}$ & single & $\mathrm{C}$ & single & $\mathrm{C}$ & single \\
\hline 89 & $\mathrm{C}$ & $\mathrm{C}$ & single & $\mathrm{C}$ & single & $\mathrm{H}$ & single & $\mathrm{H}$ & single \\
\hline 90 & $\mathrm{C}$ & $\mathrm{C}$ (aromatic) & single & $\mathrm{O}$ & double & $\mathrm{H}$ & single & - & - \\
\hline 91 & $\mathrm{C}$ & $\mathrm{C}$ & double & $\mathrm{C}$ (aromatic) & single & $\mathrm{C}$ (aromatic) & single & - & - \\
\hline 92 & $\mathrm{C}$ & $\mathrm{C}$ & single & $\mathrm{O}$ & single & $\mathrm{H}$ & single & $\mathrm{H}$ & single \\
\hline 93 & $\mathrm{~N}$ (aromatic) & $\mathrm{C}$ (aromatic) & aromatic & $\mathrm{N}$ (aromatic) & aromatic & No & - & - & - \\
\hline 94 & $\mathrm{C}$ (aromatic) & $\mathrm{C}$ & double & $\mathrm{C}$ (aromatic) & aromatic & $\mathrm{C}($ aromatic $)$ & aromatic & - & - \\
\hline 95 & $\mathrm{C}$ (aromatic) & $\mathrm{C}$ (aromatic) & aromatic & $\mathrm{C}$ (aromatic) & aromatic & S & double & - & - \\
\hline 96 & S & $\mathrm{C}$ & double & $\mathrm{C}$ (aromatic) & double & No & - & - & - \\
\hline 97 & $\mathrm{C}$ & $\mathrm{C}$ & single & $\mathrm{C}$ (aromatic) & double & $\mathrm{H}$ & single & - & - \\
\hline 98 & $\mathrm{C}$ & $\mathrm{C}$ (aromatic) & single & $\mathrm{C}$ (aromatic) & single & S & double & - & - \\
\hline 99 & $\mathrm{C}$ & $\mathrm{C}$ (aromatic) & single & S & double & $\mathrm{H}$ & single & - & - \\
\hline 100 & $\mathrm{C}$ (aromatic) & $\mathrm{C}$ (aromatic) & single & $\mathrm{C}$ (aromatic) & aromatic & $\mathrm{O}$ (aromatic) & aromatic & - & - \\
\hline 101 & $\mathrm{C}$ (aromatic) & $\mathrm{C}$ & single & $\mathrm{C}$ (aromatic) & aromatic & $\mathrm{O}$ (aromatic) & aromatic & - & - \\
\hline 102 & $\mathrm{O}$ (aromatic) & $\mathrm{C}$ (aromatic) & aromatic & $\mathrm{C}$ (aromatic) & aromatic & No & - & - & - \\
\hline 103 & S & $\mathrm{C}$ & single & $\mathrm{C}$ (aromatic) & single & No & - & - & - \\
\hline 104 & $\mathrm{~N}$ (aromatic) & $\mathrm{C}$ (aromatic) & aromatic & $\mathrm{O}$ (aromatic) & aromatic & No & - & - & - \\
\hline 105 & $\mathrm{O}$ (aromatic) & $\mathrm{N}$ (aromatic) & aromatic & $\mathrm{N}$ (aromatic) & aromatic & No & - & - & - \\
\hline 106 & $\mathrm{C}($ aromatic $)$ & $\mathrm{C}$ (aromatic) & single & $\mathrm{N}$ (aromatic) & aromatic & $\mathrm{N}$ (aromatic) & aromatic & - & - \\
\hline 107 & $\mathrm{C}$ & $\mathrm{C}$ & single & $\mathrm{C}$ (aromatic) & single & $\mathrm{H}$ & single & $\mathrm{N}$ & single \\
\hline 108 & $\mathrm{~N}$ & $\mathrm{C}$ & single & $\mathrm{C}$ (aromatic) & double & No & - & - & - \\
\hline 109 & $\mathrm{C}$ (aromatic) & $\mathrm{C}$ (aromatic) & aromatic & $\mathrm{C}$ (aromatic) & aromatic & $\mathrm{N}$ & double & - & - \\
\hline 110 & $\mathrm{C}$ & $\mathrm{C}$ (aromatic) & single & S & single & $\mathrm{N}$ & double & - & - \\
\hline 111 & $\mathrm{~N}$ & $\mathrm{C}$ & single & $\mathrm{C}$ & double & No & - & - & - \\
\hline 112 & $\mathrm{C}$ & $\mathrm{C}$ & single & S & single & $\mathrm{H}$ & single & $\mathrm{N}$ & single \\
\hline 113 & $\mathrm{C}$ & $\mathrm{C}$ & double & $S$ & single & $\mathrm{S}$ & single & - & - \\
\hline 114 & $\mathrm{C}($ aromatic $)$ & $\mathrm{C}$ (aromatic) & aromatic & $\mathrm{C}($ aromatic $)$ & aromatic & S & single & - & - \\
\hline 115 & $\mathrm{C}$ (aromatic) & $\mathrm{C}$ (aromatic) & aromatic & $\mathrm{S}$ (aromatic) & aromatic & $\mathrm{Si}$ & single & - & - \\
\hline 116 & $\mathrm{Si}$ & $\mathrm{C}$ (aromatic) & single & $\mathrm{C}($ aromatic $)$ & single & $\mathrm{C}($ aromatic $)$ & single & $\mathrm{C}$ (aromatic) & single \\
\hline 117 & $\mathrm{C}($ aromatic $)$ & $\mathrm{C}$ (aromatic) & aromatic & $\mathrm{C}$ (aromatic) & aromatic & $\mathrm{O}$ (aromatic) & aromatic & - & - \\
\hline 118 & $\mathrm{C}$ & $\mathrm{C}$ & single & $\mathrm{C}$ (aromatic) & single & F & single & $\mathrm{F}$ & single \\
\hline 119 & $\mathrm{C}$ & $\mathrm{C}$ & single & $\mathrm{C}$ & single & $\mathrm{F}$ & single & $\mathrm{F}$ & single \\
\hline 120 & $\mathrm{C}$ & $\mathrm{C}$ & single & $\mathrm{F}$ & single & $\mathrm{F}$ & single & $\mathrm{F}$ & single \\
\hline 121 & $\mathrm{O}$ & $\mathrm{C}$ (aromatic) & single & $\mathrm{H}$ & single & No & - & - & - \\
\hline 122 & $\mathrm{~N}$ (aromatic) & $\mathrm{C}$ & single & $\mathrm{N}$ (aromatic) & aromatic & $\mathrm{N}$ (aromatic) & aromatic & - & - \\
\hline 123 & $\mathrm{C}$ & $\mathrm{C}$ (aromatic) & single & $\mathrm{C}($ aromatic $)$ & single & $\mathrm{C}($ aromatic) & single & $\mathrm{H}$ & single \\
\hline 124 & $\mathrm{C}$ & $\mathrm{C}$ & single & $\mathrm{C}$ & single & $\mathrm{C}$ (aromatic) & single & $\mathrm{H}$ & single \\
\hline 125 & $\mathrm{C}$ & $\mathrm{C}$ & single & $\mathrm{C}$ (aromatic) & single & $\mathrm{H}$ & single & $\mathrm{H}$ & single \\
\hline 126 & $\mathrm{C}$ & $\mathrm{O}$ & single & $\mathrm{O}$ & double & $\mathrm{N}$ & single & - & - \\
\hline 127 & $\mathrm{C}$ & $\mathrm{C}$ & single & $\mathrm{C}$ & single & $\mathrm{C}$ (aromatic) & single & $\mathrm{O}$ & single \\
\hline 128 & $\mathrm{C}($ aromatic $)$ & $\mathrm{C}$ (aromatic) & aromatic & $\mathrm{H}$ & single & $\mathrm{O}$ (aromatic) & aromatic & - & - \\
\hline 129 & $\mathrm{~N}$ & $\mathrm{C}$ & single & $\mathrm{C}$ (aromatic) & single & $\mathrm{C}($ aromatic) & single & - & - \\
\hline 130 & S & $\mathrm{C}$ (aromatic) & single & $\mathrm{C}$ (aromatic) & single & No & - & - & - \\
\hline
\end{tabular}




\begin{tabular}{llcccccccc}
131 & $\mathrm{C}$ & $\mathrm{C}($ aromatic $)$ & single & $\mathrm{N}$ & triple & $\mathrm{No}$ & - & - & - \\
132 & $\mathrm{C}$ & $\mathrm{O}$ & double & $\mathrm{N}$ & single & $\mathrm{N}$ & single & - & - \\
133 & $\mathrm{C}$ & $\mathrm{C}($ aromatic $)$ & single & $\mathrm{C}$ (aromatic) & single & $\mathrm{O}$ & double & - & - \\
\hline
\end{tabular}

\section{DETAILED INFORMATION ABOUT BUILDING BLOCKS}

For the molecules in CEPDB, they are based on 26 building blocks and bonding rules shown in Fig. S2. As illustrated in Fig. S2, the 26 building blocks consist of 8 element species: C, H, O, N, S, Si, F and three chemical bond types: aromatic bond, single bond and double bond.
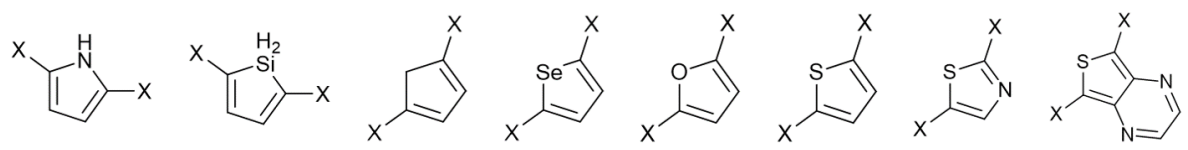<smiles>[X]c1cccc2c([X])cccc12</smiles><smiles>[X]c1cnc([X])c2nsnc12</smiles><smiles>[X]c1ccc([X])c2c[nH]cc12</smiles><smiles></smiles><smiles>[X]c1ccc([X])c2[nH]ccc12</smiles><smiles>[Y]c1ccc([X])c2cscc12</smiles><smiles>[X]c1sc([X])c2c3ccccc3c3ccccc3c12</smiles><smiles>[X]c1ccc([X])cc1</smiles><smiles>[X]c1ncncn1</smiles><smiles>[X]c1sc([X])c2[se]ccc12</smiles><smiles>[X]c1sc([X])c2[nH]ccc12</smiles><smiles>[X]c1sc([X])c2sccc12</smiles><smiles>[X]c1sc([X])c2occc12</smiles><smiles>[X]c1sc([X])c2c1C=CC2</smiles>

Figure S2. The 26 building blocks used for generating the organic compounds in CEPDB. The $\mathrm{X}$ atoms represent chemical handles, i.e, the reactive sites in the generation process.

\section{BFS ALGORITHMS OF PRE-ENCODING}

Breadth-first search (BFS) algorithm has been applied in this paper to generate the molecular descriptor. It is a common algorithm for traversing graph or tree data structures by exploring all the nodes at the present layer before moving on to the nodes at the next year. Here, a four-layer binary tree (Fig. S3) is regard as a specific example to demonstrate how BFS works. First, we select the node 1 as the source node, and then we keep exploring down the branches in an ordered fashion i.e. from 1 to 2 to 3 where the layer completes. The final result of traversing the tree is the route 1-2-3-45-6-7-8-9.

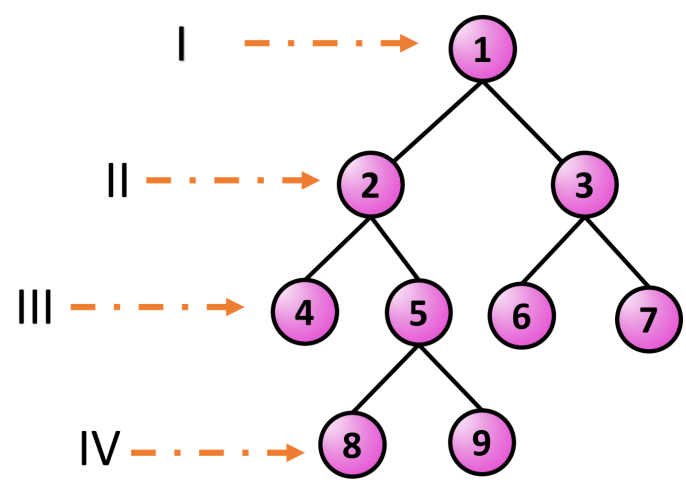

Figure S3. BFS algorithm 
Table S4. Prediction accuracy of the testing set

\begin{tabular}{|c|c|c|c|c|c|c|c|}
\hline Index & Measure & MSE & $R^{2}$ & Index & Measure & MSE & $R^{2}$ \\
\hline 1 & $\mathrm{~g}$-FSI + Bi-LSTM + attention & 0.12 & 0.98 & 2 & $\mathrm{~g}-\mathrm{FSI}(\mathrm{DFS})+\mathrm{Bi}-\mathrm{LSTM}+$ attention & 0.29 & 0.95 \\
\hline 3 & g-FSI(NR) + Bi-LSTM + attention & 0.30 & 0.95 & & & & \\
\hline
\end{tabular}

\section{COMPETITIVENESS}

There are also some other algorithms for exploring graphs and trees such as depth-first search (DFS), which is as common as BFS. To demonstrated the effect of the BFS in the molecular generating process, we also apply the DFS algorithm to generate the molecular descriptor (g-FSI(DFS)). At the same time, the default descriptor generated by rdkit without rearranging the molecular sequence (g-FSI(NR) is also taken into account.

In Fig. S4, we give an example (SMILES:c1cc2c3nsnc3c3c4[SiH2]C( $=\mathrm{Cc} 4 \mathrm{c} 4 \mathrm{c}[\mathrm{nH}] \mathrm{cc} 4 \mathrm{c} 3 \mathrm{c} 2 \mathrm{o} 1) \mathrm{c} 1 \mathrm{cccc} 2 \mathrm{c}[\mathrm{nH}] \mathrm{cc} 12)$ to illustrate the results of the three descriptors. As is shown in Fig. S4, the final results of g-FSI(NR) (a) and g-FSI(DFS) are similar, and so are the results of property prediction with the two descriptors, which are shown in table S4. Furthermore, the two descriptors perform worse than that with BFS algorithm also.

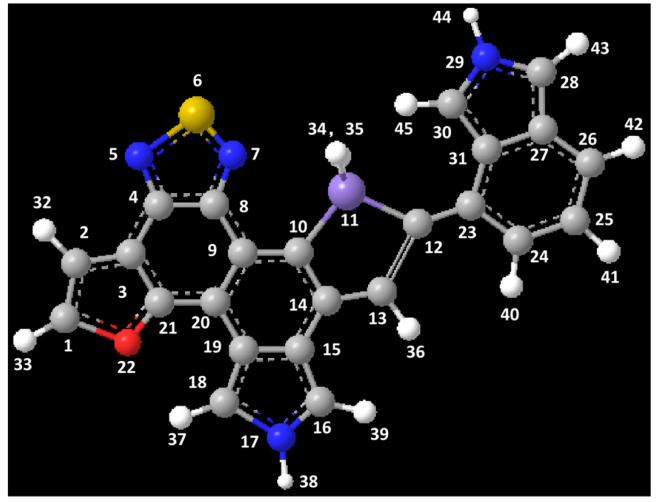

(a)

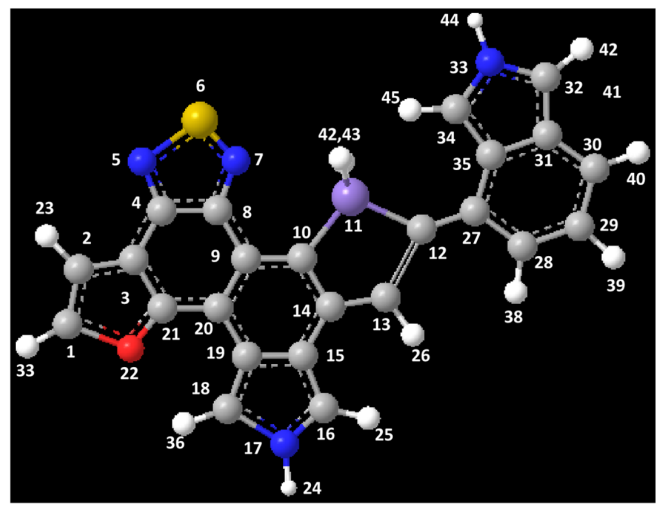

(b)

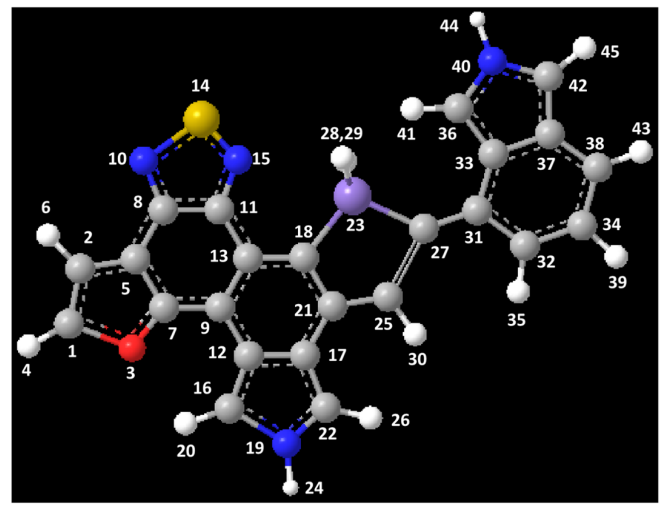

(c)

Figure S4. The results of traversing a given compound: (a) g-FSI(NR) (b) g-FSI(DFS) (C) g-FSI 